\title{
The Impact of Stochastic and Deterministic Sounds on Visual, Tactile and Proprioceptive Modalities
}

\author{
J.E. Lugo, R. Doti and J. Faubert \\ Visual Psychophysics and Perception Laboratory, \\ School of Optometry, University of Montreal, \\ C.P. 6128 succ. Centre Ville, Montréal, \\ Quebéc, H3C3J7 \\ Canada
}

\section{Introduction}

Stimulus localization and particularly directional hearing can be considered as methods for investigating neural activity and they have proven to be useful tools for research in physiology and psychology. Human directional hearing techniques have been reflected upon way back by Von Békésy in Austrian forests [1]. For example, he observed that some of the roads took a perfectly straight course through deep, dark woods. He could not imagine how such straight roads had been cut through the forest when the usual optical methods used by road surveyors would seem to be useless in this case. Further some of these roads were very old and probably built before the introduction of the theodolite. Many of these roads were laid out by an acoustic method. How did they do it? A man stationed at the starting point noted the direction of the sound produced by someone at the other end blowing a horn. The first man then walked toward the sound source, marking the threes on the way. It turned out that this method produced a straight line from start to finish [1]. From this observation Békésy was motivated to perform a series of studies on stimuli localization not limited to hearing but also to vibration sensations on the skin, electrical pulses on the tongue and odors through the nose as well. Strikingly, his results showed an underlying ubiquitous mechanism present in the different stimuli localization modalities. For instance, the effect on localization of the time delay between two stimuli on the skin, the tongue, the two nostrils in the nose and the two ears, presented the same dynamics [2-4]. These results were quite exciting because it showed that, in humans, the senses work similarly for stimuli localization although the basic underlying neural pathways are not the same.

It was this kind of general principle on stimuli localization that motivated us in the search for more general principles related to how senses interact to generate multisensory perceptions but with a special emphasis on auditory stimulation. This is known as multisensory integration and its study is very important because it is the foundation of how humans bind all the information coming from the senses to generate a coherent percept. We began by studying something that we called cross-modal stochastic resonance. This consists 
in the concurrence of a threshold, a subthreshold stimulus present in one sense and noise at different amplitudes entering through another sense. What we found was that the same auditory noise can enhance the sensitivity of tactile, visual and propioceptive system responses to weak signals. Specifically, we showed that the effective auditory noise significantly increased tactile sensations of the finger, decreased luminance and contrast visual thresholds and significantly changed EMG recordings of the leg muscles during posture maintenance [5]. We also found that in all the cases the interactions follow the same sort of physical dynamics. Moreover, we unveil that the same result is obtained if we use auditory deterministic sounds instead of auditory noise [6] to enhance tactile sensations. We further demonstrated that we could use tactile noise and enhance visual detection [7] or use visual deterministic signals to enhance tactile detection [6]. These surprising results guided us to propose that these multisensory integration interactions can be explained under the same general principle that we call the Fulcrum principle.

In this chapter we present material emerging from our own research experience concerning human perception in general with emphasis in auditory interactions. We introduce in an accessible way a non-linear mathematical model supporting our hypothesis, and we provide experimental results and conclusions. We also propose that the Fulcrum principle may have numerous implications in a number of neurobiological alterations such as autism, aging and age-related neurodegenerative disorders and ADHD. We conclude by presenting to the readers with what we consider could be the next hurdles in this area, and the main points that we think should be emphasized in future work.

\section{Multisensory Integration: MI}

A general description: $\mathrm{MI}$ is a non linear process that binds information from all the participating sensory stimuli. The original approach shows that MI results from the brain's capacity for integrating information originating from more than a single sensory stimulus. Here we would like to present the two stimuli conditions allowing us to introduce the mathematical model.

The first aspect involves the concept of Signal Coherence, and the second important aspect is the Sense Threshold for those signals [8]. Coherence is intended to be the propriety that gives the signal a continuous and repetitive harmonic shape. A signal involves the concept of evolution in the time domain, harmonic shape implies the same amplitude at regular time intervals, and very importantly, the same amount of energy transferred per unit of time [9]. If we have more than one stimulus applied to a big surface interface, we can split this concept in two: Temporal Coherence (frequency) and Spatial Coherence (front- wave)

Temporal Coherence: when we consider the coexistence of more than one stimulus signal, the coherence associated with this compound stimulus is the correlation (proportional correspondence) between the evolutions in the time domain for both signals (together). When the signals are periodic this represents the same frequency spectrum content and results in the same bandwidth (BW) [10]. In the case of a pure tone, we would have only one frequency component in the signal spectrum.

Spatial Coherence: if for a fixed point in space along the signals pass the superposition of these simultaneous signals presents Temporal Coherence, we say that signals have spatial coherence. The front-wave of this compound signal preserves the shape along its pass (when traveling along an ideal non dispersive mean). 

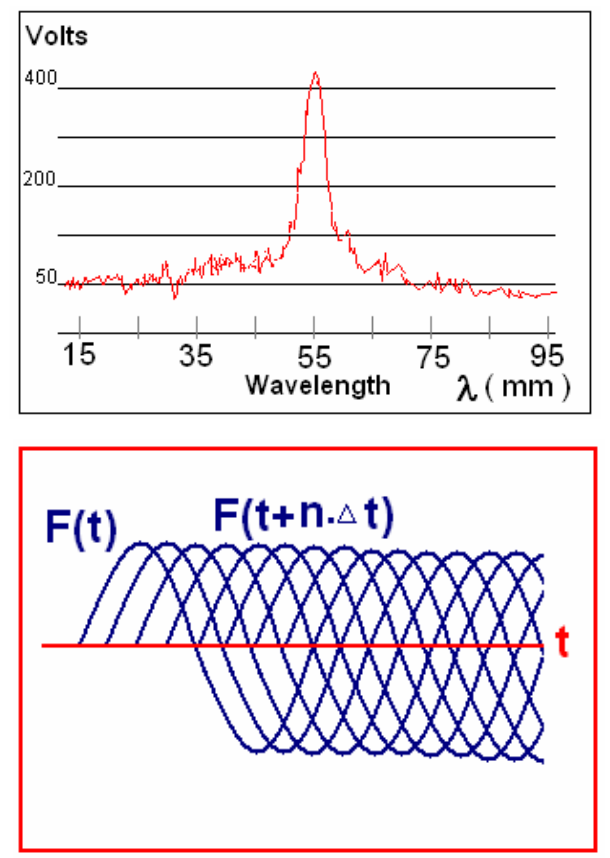

Examples of periodic signals

So, depending on the intensity and characteristic of the stimulus signal we can have different situations. For instance, for a given perceptual threshold level we can have: suprathreshold (perceived signal), or sub-threshold (not perceived) stimuli. Depending on the stability and consistency of the signal stimuli we can have deterministic signals (coherent or not) or stochastic signals.

Periodic signals means a fixed

frequency spectrum content or a fixed

bandwidth (BW).For a pure tone, we have a narrow frequency spectrum

Deterministic signals always present a limited bandwidth or a repetitive pattern. They can be described and recreated without error along the time domain. We know the evolution of the instantaneous energy transferred trough these signals. 
Stochastic signals represent a random pattern and a very large bandwidth. We can establish the limits of their characteristics (amplitude or BW), but we do not know in advance their evolution along the time domain. We know the mean energy transferred trough these signals. A good example of a Stochastic Signal is White Noise [11].
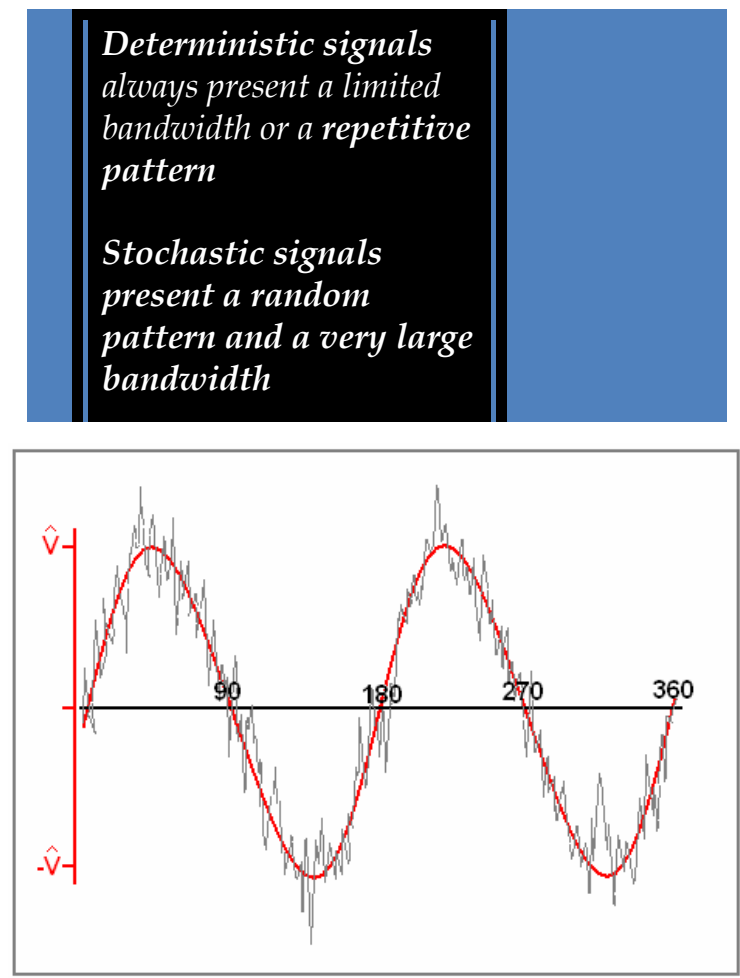

Example of a deterministic signal with noise

Because of the random instantaneous frequency content compared with a pure tone, we call it NOISE. As its Frequency Spectrum extends from zero Hz to infinite, we call it WHITE (in analogy with the visible spectrum and the eyes perception of the white light).

\section{The Inverse-effectiveness law}

So far we defined the MI as the complex way in which our brain binds the different sensory stimuli that contributes to create a phantom image of the real world outside its perceptual limits. This image is the only reality we have. Researchers tried to define the human sensory stimulus span from threshold to ceiling. They tested humans applying deterministic stimuli signals to the different senses. This generated normalized thresholds for auditory, tactile, visual, etc.

Here we find the first cue in reference to MI: it was determined that if two weak (close to threshold level) stimuli are applied together, the presence of the additional stimulus facilitates perception. And this happens for an elastic temporal coincidence. But, this perceptual improvement is not possible if one of the stimuli is clearly supra-threshold. This 
is known as: Inverse-Effectiveness Law [12]. This means that perceptual enhancement takes place trough the MI mechanism when we apply: weak, supra-threshold, deterministic and coincident signals to the subject. However, there is an MI phenomenon that cannot be described by the inverse-effectiveness rule: cross-modal SR.

\section{Stochastic resonance}

Stochastic resonance (SR) [13] is a nonlinear phenomenon whereby the addition of noise can improve the detection of weak stimuli. An optimal amount of added noise results in the maximum enhancement, whereas further increases in noise intensity only degrade detection or information content. The phenomenon does not occur in linear systems, where the addition of noise to either the system or the stimulus only degrades the measures of signal quality. The SR phenomenon was thought to exist only in stochastic, nonlinear, dynamical systems but it also exists in another form referred to as 'threshold SR' or 'non-dynamical $\mathrm{SR}^{\prime}$. This form of stochastic resonance results from the concurrence of a threshold, a subthreshold stimulus, and noise. These ingredients are omnipresent in nature as well as in a variety of man-made systems, which accounts for the observation of SR in many fields and conditions. The SR signature is that the signal-to-noise ratio, which is proportional to the system's sensitivity, is an inverted U-like function of different noise levels. That is, the signal-to-noise ratio first is enhanced by the noise up to a maximum and then lessened. The SR phenomenon has been shown to occur in different macro [14], micro[15] and nano physical systems [16]. From the cyclic recurrence of ice ages, bistable ring lasers, electronic circuits, superconducting quantum interference devices (SQUIDs) and neurophysiological systems [17] such as receptors in animals. Several studies have suggested that the higher central nervous system might utilize the noise to enhance sensory information [13]. SR studies in humans can be divided in unimodal SR (signal and noise enter the same sense) $[18,19]$, central SR (signal and noise enters in similar local receptors and later mix in the cortex) [20] and behavioral SR (similar to central SR but its effect is observed in one sense and then enacted in the behavior of the subjects) [21]. Before the SR principle was proposed, Harper [22] discovered what we currently would call crossmodal stochastic resonance while studying the effect of auditory white noise on sensitivity to visual flicker. Recently a similar result [23] has been found where auditory noise produces SR when subthreshold luminance stimuli are present. However what has not been explored is the extension of these interactions in humans. New results show that the noise induces large scale phase synchronization of human-brain activity associated with behavioral SR [24]. It is shown that both detection of weak visual signals to the right eye and phase synchronization of electroencephalogram (EEG) signals from widely separated areas of the human brain are increased by addition of weak visual noise to the left eye. These results imply that noiseinduced large-scale neural synchronization may play a significant role in information transmission in the brain. Interestingly SR can be seen as a synchronization-like phenomenon between two energy states of a physical system for example [25]. Furthermore, the synchronization-like phenomenon plays a key role in the enhancement of the signal-tonoise ratio in SR. Therefore, we can hypothesized that if the noise induced large scale phase synchronization in different areas of the cortex and peripheral systems with dynamics similar to SR, the crossmodal SR would be a ubiquitous phenomenon in humans because it involves different cortical areas and peripheral systems. Consequently under the same auditory noise conditions, the crossmodal SR should be present among tactile, visual and proprioceptive sensory systems, for instance. 


\section{Facilitating and excitatory stimulus}

In order to outline a synoptic scheme that represents the basis of some experiments that we have performed, we introduce another two concepts. First, Excitatory Stimulus: signal applied to the sense that we want to study. Second, Facilitating Stimulus: signal applied simultaneously to the same subject, intended to trigger the MI mechanism in a way that facilitates the perception of the Excitatory Stimulus. When both, facilitating and excitatory signals act as stimuli of the same sense (auditory, tactile, visual stimulus, etc) we have Unimodal Interactions (U.M). When each one of these signals act in different senses (for instance excitatory: tactile; and facilitating: auditory) we have Cross-modal Interactions (C.M). Either of the precedent cases are part of the general Multi-modal Interactions model.

\section{Crossmodal interactions paradigms and the sensory threshold enhancement}

On the basis of what was presented so far, it is possible to combine those elements to create the experiments that allow us to explore human perception and outline a plausible model. All of them allow a positive response from the subject under test, by the action of the facilitating stimulus, when the excitatory stimulus is Sub threshold. This means an improvement of the human perception. Examples of multimodal interactions that have been tested so far are:

\section{Excitatory: Tactile - Deterministic- threshold}

Facilitating: Auditory or Visual -Deterministic - threshold

2. Excitatory: Tactile - Deterministic- Sub threshold

Facilitating: Auditory - Stochastic - Supra threshold

3. Excitatory: Visual - Deterministic-Sub threshold

Facilitating: Auditory - Stochastic - Supra threshold

4. Excitatory: Propioception - Deterministic-Sub threshold

Facilitating: Auditory - Stochastic - Supra threshold

5. Excitatory: Visual - Deterministic- Sub threshold

Facilitating: Tactile - Stochastic - Supra threshold

6. Excitatory: Tactile - Deterministic- Sub threshold

Facilitating: Auditory - Deterministic - Supra threshold

7. Excitatory: Tactile - Deterministic-Sub threshold

Facilitating: Visual - Deterministic - Supra threshold
E:T-D- T
F: AoV-D-T
E:T-D-ST
F: A-S- SST
E:V-D-ST
F: A-S- SST
E:P-D-ST
F: A-S- SST
E:V-D-ST
F: T-S- SST
E:T-D-ST
F: A-D- SST
E:T-D-ST
F: V-D- SST

We observe that $\mathbf{1}$ is a cross modal example of the Inverse Effectiveness Law (IEL). These kinds of examples have been studied massively and they are well documented on the literature [12]. 2 to 5 belong to the Multi modal Stochastic Resonance (MmSR) and $\mathbf{6}$ and $\mathbf{7}$ belong to the Multi modal Deterministic Resonance (MmDR). In what follows we will explain more in detail these multimodal interactions.

Excitatory: Tactile - Deterministic- Sub threshold

Facilitating: Auditory - Stochastic - Supra threshold

In the first series of experiments we studied the effects of auditory noise on tactile sensations in three subjects. Tactile vibrations were delivered to the middle finger of the right hand of the subjects at a frequency of $100 \mathrm{~Hz}$ and were asked to report the tactile sensation. If they felt the signal they had to click on a yes button or on a no button otherwise (yes-no paradigm). Each subject was tested twice for every auditory noise and baseline condition. In 
all the experiments were the facilitating signal was auditory the normalized thresholds were computed as follows: once the absolute threshold was obtained for different auditory noise conditions, their values were divided by the absolute threshold measured for the baseline condition. Figure 1 (left column) shows the normalized tactile thresholds for three subjects and it is clear that, as the noise level increased, the threshold decreased reaching a minimum and then increased in a typical SR signature fashion. In general we found that the subject's minimum peaks are not always localized at a specific noise level but within a band centered at $69 \pm 7 \mathrm{dBSPL}$. Can the above results be explained only on the bases of SR theory? Can one potentially rule out an explanation based on attention/arousal? If the noise creates a more interesting/arousing condition than the baseline condition, all neural systems could be correspondingly more excitable, not because the noise facilitates a resonance like behaviour but because the auditory noise nonspecifically boosts neural excitability. However, the Yerkes- Dodson law demonstrates an empirical relationship between arousal and performance [26]. Such relationship is task dependent. For instance, in a simple task the relationship between arousal and performance is linear and only in a difficult task this relationship becomes curvilinear (inverted u-shape similar to SR). Since a yes-no procedure with vibration thresholds would be considered a very simple task, we would not expect an inverted ushape between the noise level and tactile sensitivity if the mechanism involved in these interactions was only arousal. That was not the case as Fig. 1 clearly shows a curvilinear relationship. In order to further explore the notion of possible attention effects we performed an additional experiment on sixteen subjects where we used two different auditory stimuli plus the baseline condition. One stimulus was a specific auditory noise condition as described above, and another was a 3D-like sound. Both sounds had an intensity of $69 \mathrm{dBSPL}$ and the 3D sound contained frequencies in a similar range as the auditory noise (between $100 \mathrm{~Hz}$ up to $19 \mathrm{kHz}$ ). The 3D sound gave the impression of very close movements near, up and down, and around the subjects' head resulting in a very strong attention getting sound sequence. If our previous results were only a result of attention modulation created by the sound intensity, we should expect that for, the 3D auditory condition, the tactile thresholds would be lower in most people because this sequence had strong attention modulation properties and the noise level we chose was the same as the averaged peak noise level we measured in the first experiment that generated the lowest tactile thresholds. An alternative hypothesis is that this attention-producing stimulus would not influence or maybe even hinder tactile performance. On the other hand, we did expect the auditory noise condition to generate lower tactile thresholds given that we chose the averaged peak noise level that generated the lowest thresholds in the previous experiment. Each subject was tested twice for every condition in randomized order. Fig. 1 (right column, top) shows the normalized tactile thresholds for the 3D sound and baseline conditions. Eight subjects augmented significantly their thresholds comparatively to baseline condition, four subjects lessened theirs thresholds and in other four subjects the threshold values remained unchanged. Fig. 1 (right column, middle) shows the normalized tactile thresholds for the auditory noise and baseline condition. Twelve subjects significantly lessened their thresholds, only two subjects increased their thresholds and another two subjects had unchanged threshold values. Fig. 1 (right column, bottom) shows the group average of the normalized tactile threshold for the three conditions. The average group sensitivity increased significantly (with respect the baseline) in the presence of noise $(p<0.001)$ while no significant change was found for the 3Dlike sound $(p=0.72)$. It is clear from these experimental controls, that the noise effects on tactile sensations are not due to 

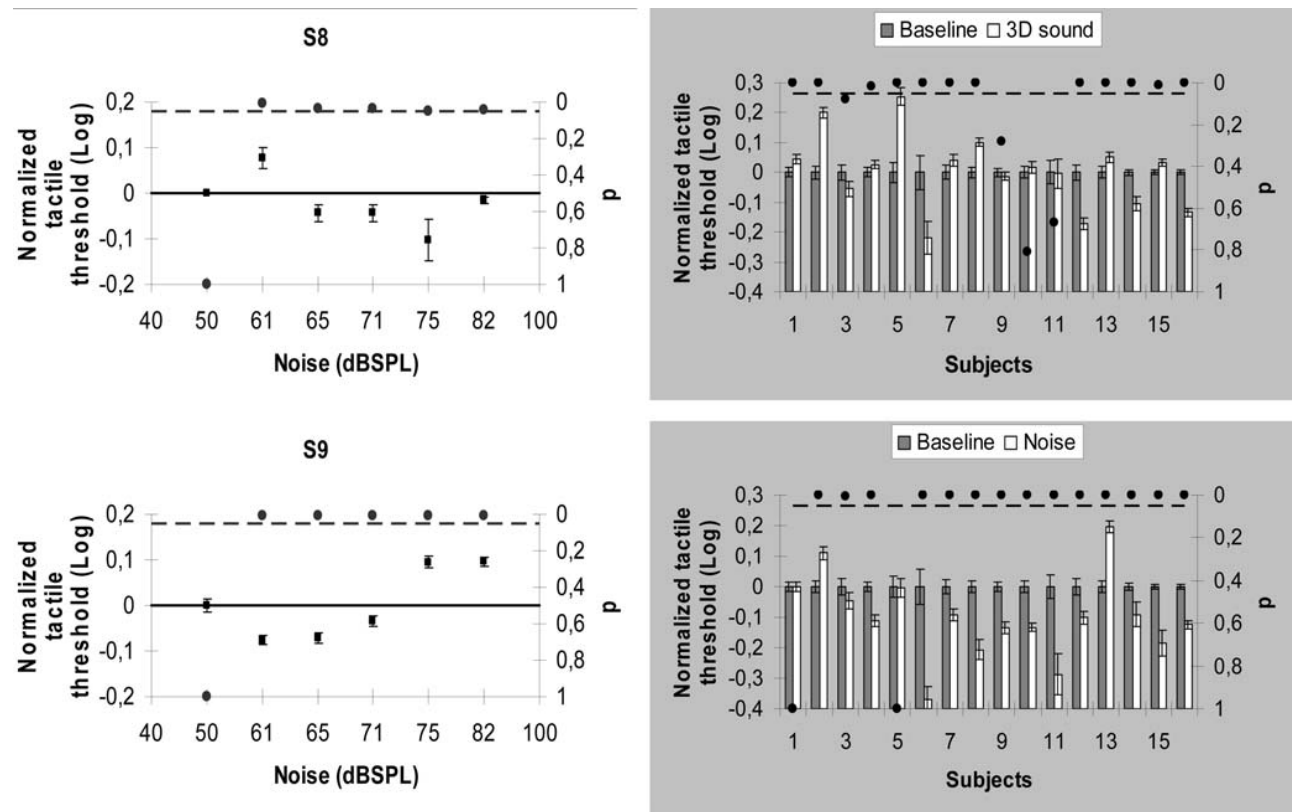

S17

Group average at $69 \mathrm{dBSPL}$
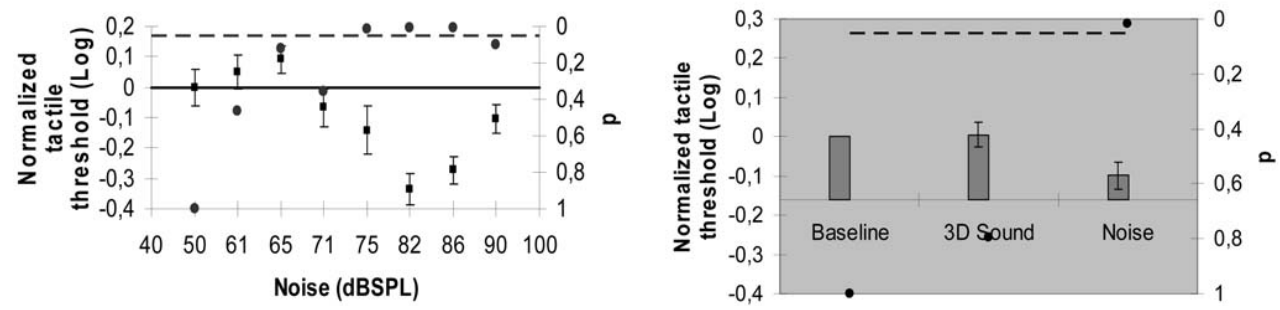

Fig. 1. Interactions between auditory noise and tactile signals. (Left column) normalized tactile threshold changes with the noise level in three subjects. (Right column, top) normalized tactile thresholds of sixteen subjects when the 3D sound level was fixed at 69 dBSPL. (Right column, middle) normalized tactile thresholds of sixteen subjects when the noise level was fixed at 69 dBSPL. (Right column, bottom) Group average results for three conditions: baseline, 3D sound and noise. The average group threshold decreased significantly in the presence of noise $(p, 0.001)$ and no significant change was found for the 3D-like sound $(p=0.72)$. In all the graphs the no-noise condition is taken as baseline; the black dots indicate pvalues (right y-axis) and the broken line represents the $5 \%$ significance level. Error bars correspond to one standard error. 

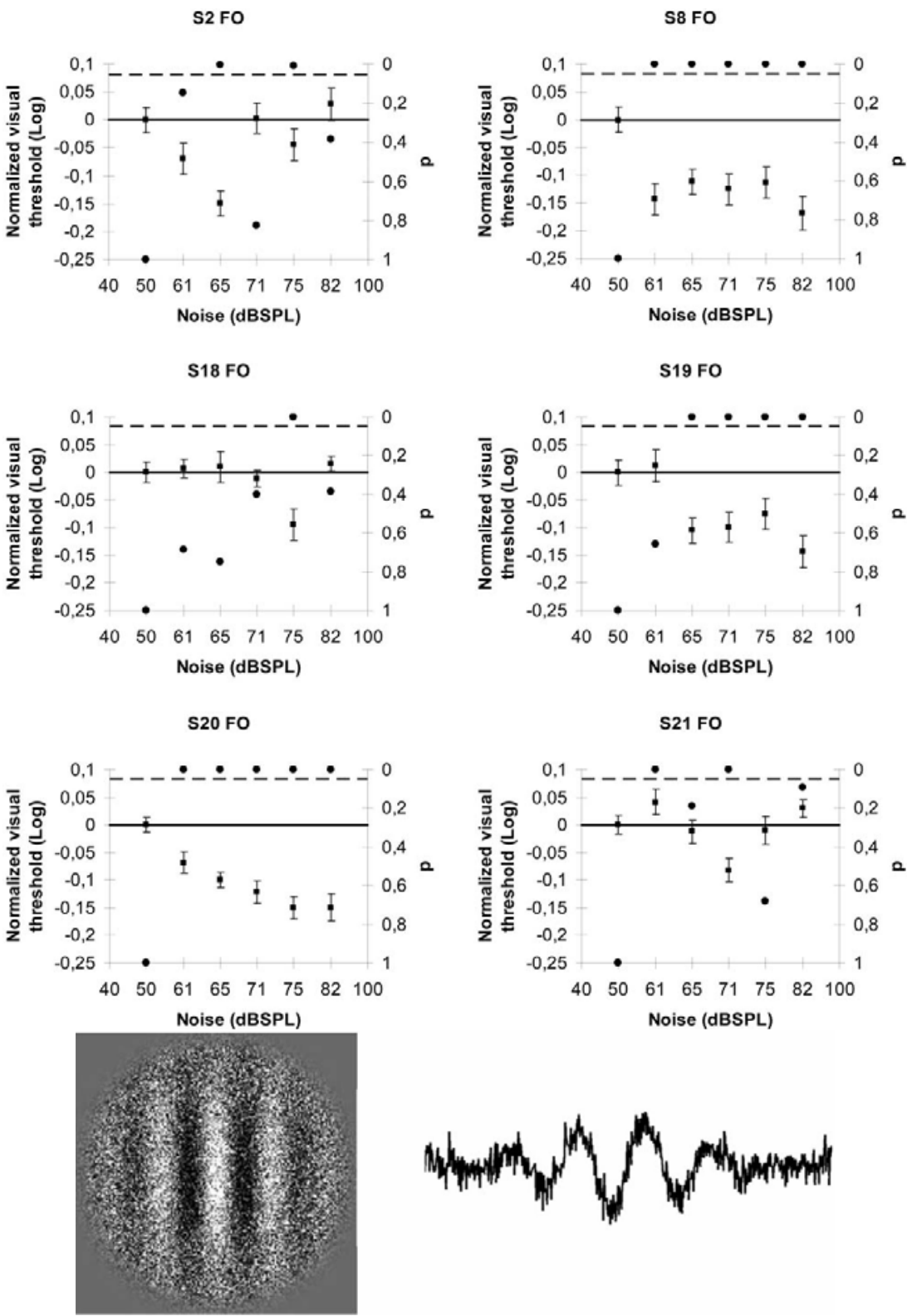

Fig. 2. Interactions between auditory noise and first order visual signals. Normalized visual threshold changes with the noise level in sixth subjects for luminance modulated (first order) stimuli. In all the graphs the no-noise condition is taken as baseline; the black dots indicate pvalues (right y-axis) and the broken line represents the 5\% significance level. Error bars correspond to one standard error. In the last row an example of the first order stimulus is displayed. 
attention/ arousal effects but result from the way the brain processes the energy (and probably the frequency) content of noise and signal.

Then we studied auditory-visual interactions. In previous work [22,23] only visual stimuli classified as first order stimuli were used. We wanted to evaluate the effect of SR on an additional visual attribute called second order processing. For first order stimuli, the local luminance spatial average varies throughout the stimulus while the local contrast remains constant. In second order stimuli, known to be processed by separate mechanisms and assumed to be more complex to process, the local spatial luminance average remains constant but the local contrast varies throughout the stimulus [27,28].

\section{Excitatory: Visual - Deterministic- Sub threshold}

Facilitating: Auditory - Stochastic - Supra threshold

\section{E:V-D-ST}

\section{F: A-S- SST}

In the second series of experiments, we studied whether auditory noise can facilitate luminance-modulated (first order) stimuli detection in six subjects. To evaluate visual thresholds, we used a two-alternative forced choice paradigm. In a two-alternative forced choice paradigm, the subject is presented two choices and must pick one (even if the observer thinks he/she did not see the stimulus), which produces a more stringent control of observer criteria than a yes/no response. Here the observers had to discriminate between vertical or horizontal luminance-modulated stimuli (LM) defined sinusoidal gratings $[27,28]$. We measured the LM thresholds for six auditory conditions (baseline plus five noise levels) in a random order. Five thresholds (5 separate staircases) were established for each condition and averaged. Fig. 2 shows the normalized visual LM thresholds for six subjects. As in our previous auditory-tactile experiments, the visual threshold profiles of the observers varied as a function of the different auditory noise levels demonstrating a typical SR function with zones of threshold values significantly different from the control condition. The SR average peak for our data was $75 \pm 3$ dBSPL for LM stimuli. Previous reports show an average value of $70 \pm 2.5 \mathrm{dBSPL}$ for visual flicker detection [22] and a value of $73.8 \pm 15.5$ dBSPL for a luminance-defined stimulus [23].

In the third series of experiments, we studied whether auditory noise can facilitate contrastmodulated (second order) stimuli detection. With the same procedure as above, the observers had to discriminate between vertical or horizontal contrast-modulated stimuli (CM) defined sinusoidal gratings [27,28]. We measured the CM thresholds for six auditory conditions (baseline plus five noise levels) in a random order. Five thresholds (5 separate staircases) were established for each condition and averaged. Fig. 3 shows examples of the normalized visual $\mathrm{CM}$ thresholds for the same six subjects. As in our previous auditoryvisual experiments, the visual CM threshold profiles of the observers varied as a function of the different auditory noise levels demonstrating a typical SR function with zones of threshold values significantly different from control. The SR average peak was found at $70 \pm 2 \mathrm{dBSPL}$ for CM stimuli. Clearly both peaks are inside the same experimental region and there is no significant difference between them meaning that within the experimental accuracy we have used both SR mechanisms are similar.

\section{Excitatory: Propioception - Deterministic- Sub threshold E:P-D-ST}

\section{Facilitating: Auditory - Stochastic - Supra threshold F: A-S- SST}

In the fourth series of experiments we evaluated electromyography (EMG) responses of the subject's leg muscles during posture maintenance with different auditory noise conditions. Recent evidence has demonstrated that tactile stimulation of the foot with noise could increase postural stability by acting on the somatosensory system and that noise can induce 

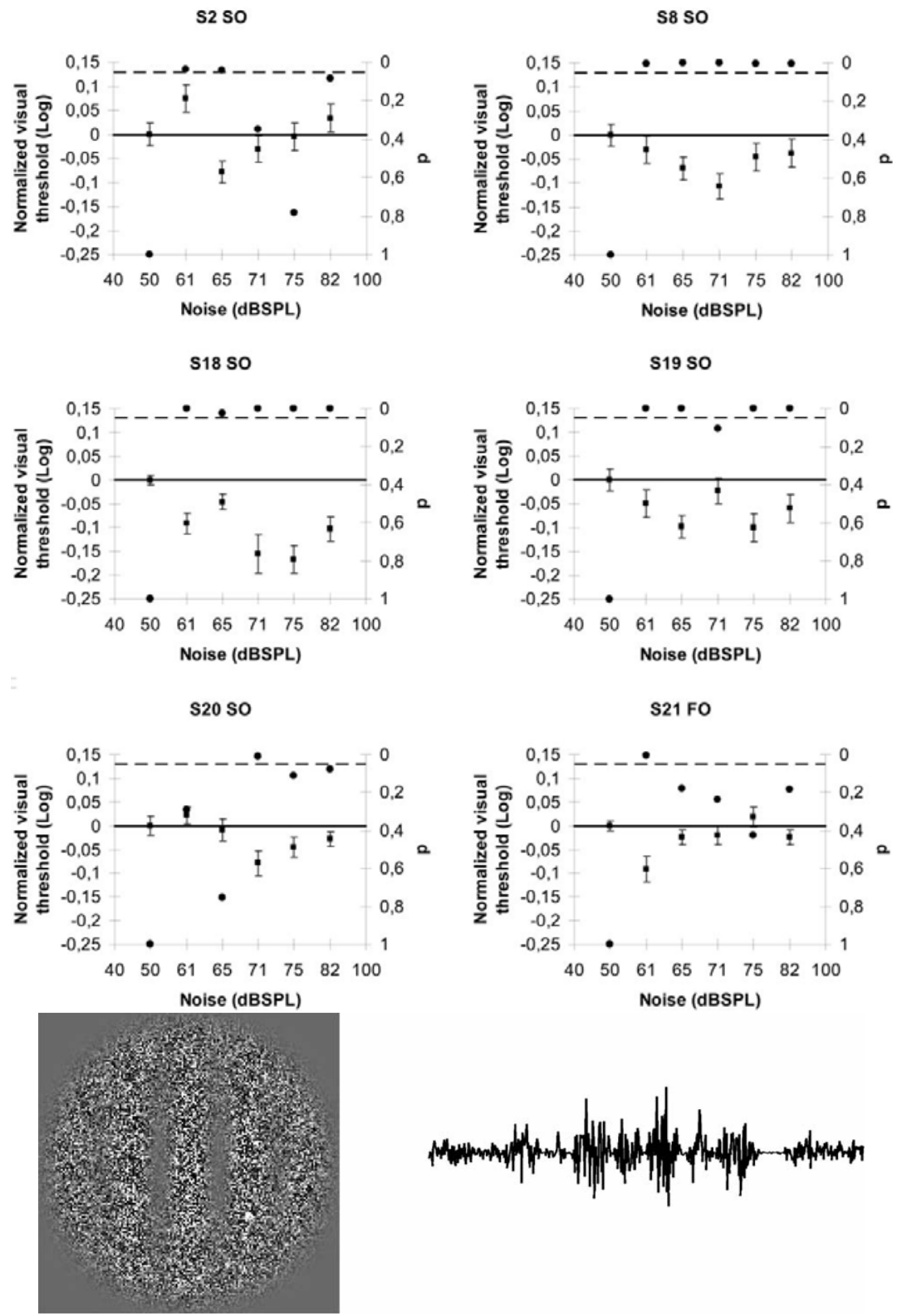

Fig. 3. Interactions between auditory noise and second order visual signals. Normalized visual threshold changes with the noise level in sixth subjects for contrast modulated (second order) stimuli. In all the graphs the no-noise condition is taken as baseline; the black dots indicate p-values (right y-axis) and the broken line represents the $5 \%$ significance level. Error bars correspond to one standard error. In the last row an example of the second order stimulus is displayed. 
transitions in human postural sway [29-31]. Four subjects were asked to stand with their feet aligned one in front of the other and touching like in a tightrope position. For all conditions (the baseline plus five noise levels) we have measured the EMG activity of each subject three times in a randomized order. In figure 4 (left column) we show the averaged EMG power spectrum density as a function of noise intensity in four subjects. The right column of figure 4 shows the normalized power of the EMG activity in the same four subjects with different noise levels and the baseline. The EMG activity refers to the muscle's activity during posture maintenance. In this context a less stable posture represents more activity of the muscles related to this task. Again the SR signature was observed by using similar noise levels as the tactile and visual experiments and surprisingly, the subject's averaged peak $74 \pm 4 \mathrm{dBSPL}$ lies in the same experimental range found in our previous experiments.
Excitatory: Visual - Deterministic - Sub threshold
E:V-D-ST
Facilitating: Tactile - Stochastic - Supra threshold
F: T-S- SST

In a sixth series of experiments, we applied different tactile noise intensity levels plus a baseline (no tactile noise) in randomized order (Figure 5) in 7 healthy subjects [7]. This randomized order of sessions assured that the observed effects are not simply due to a generalized modulation in attention/arousal. We maintained the intensity of the continuous tactile input noise constant for each session and varied it between sessions. We have measured absolute first order visual (in arbitrary units) thresholds and then normalized. Normalized visual thresholds were computed as follows: once the absolute threshold was obtained for different tactile noise conditions, their values were divided by the absolute threshold measured for the baseline condition. The experiments took place in a dark room for vision testing. The tactile noise was presented by means of a specific designed transferred signal spectrum actuator (TSSA) that converted the auditory signal spectrum energy into mechanical signal spectrum energy. The subjects held the TSSA against their right internal metacarpus. The tactile noise has a cut-off frequency around $1 \mathrm{kHz}$. We found that tactile noise also facilitated first order stimuli perception in 5 subjects similar to the auditory noise case (the tactile noise may be was out of range to show facilitation in the other two subjects).

We decided to explore if facilitating deterministic signals can induce changes on the perception in a similar fashion as in the stochastic experiments [6]. In this case we used electrical signals that were delivered to the right calf (gastrocnemius medial head) of different subjects (fig.6). With the right electrical signal amplitude, the signal was not perceived but the electrical activity in the muscle it was measurable with electromyography (EMG) electrodes. If the subjects were presented a noticeable sound or a visible pip at the same time their muscles received the electrical signal, their muscular EMG response was amplified. Furthermore, the dynamic of these interactions was similar to the precedent stochastic case. In order to obtain individual tactile thresholds the signal amplitude started out at a low level so that it could not be detected, then the amplitude was gradually increased until the subjects reported that they were aware of it. This is known as the ascending threshold. Then signal amplitude started out at a high level so that it was perfectly detected, then the amplitude was gradually decreased until the subjects reported that they were not aware of it, this is the descending threshold. The absolute threshold was the average of both thresholds. After the data were collected, the power spectral density (PSD) of each EMG measurement was obtained. To calculate the normalized PSD for each condition, $\Psi_{N}(\omega)$ (where $\omega$ is the frequency in hertz), we divided the PSD at the suprathreshold level by the corresponding PSD at the subthreshold level on each trial and then averaged across trials. 
s7

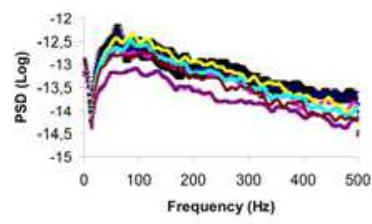

s8

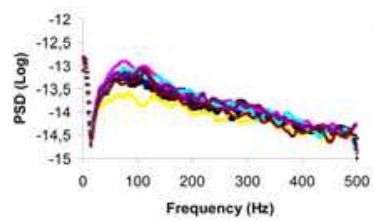

s9

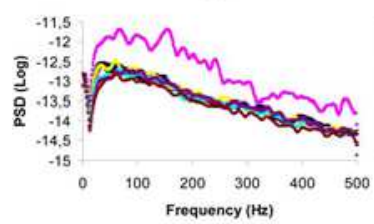

\$15

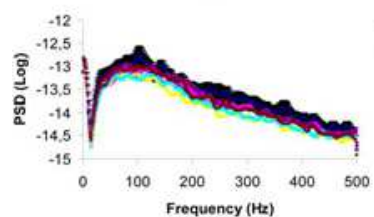

s7

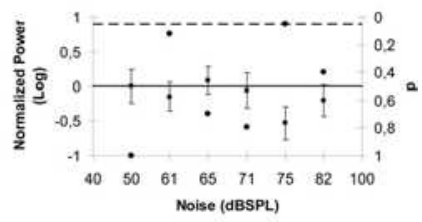

s8

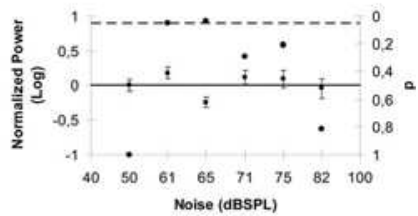

s9

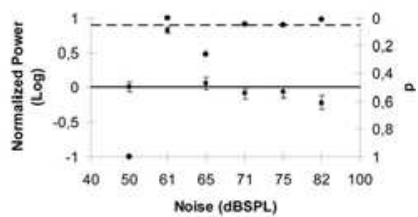

s15

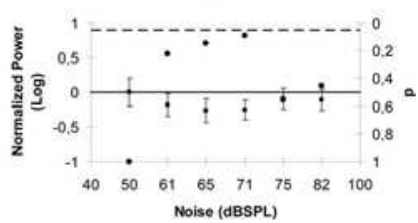

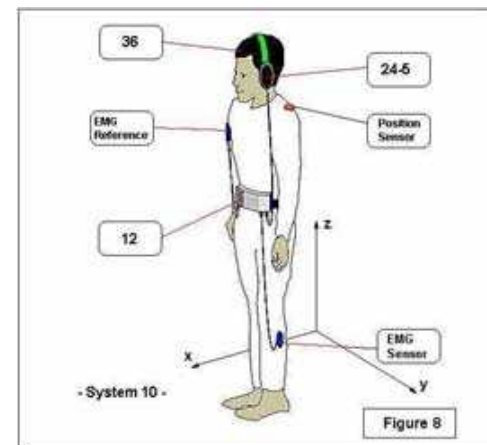

Fig. 4. Interactions between auditory noise and propioceptive signals. (Left column) average EMG power spectral densities as a function of noise level in four subjects for the tightrope posture position. For clarity only the baseline condition shows error bars (one standard error). (Right column) normalized power in four subjects. Again, the no-noise condition is taken as baseline; the black dots indicate p-values (right y-axis) and the broken line represents the 5\% significance level. Error bars correspond to one standard error. In the last row an example on how the experiments were done is displayed. 

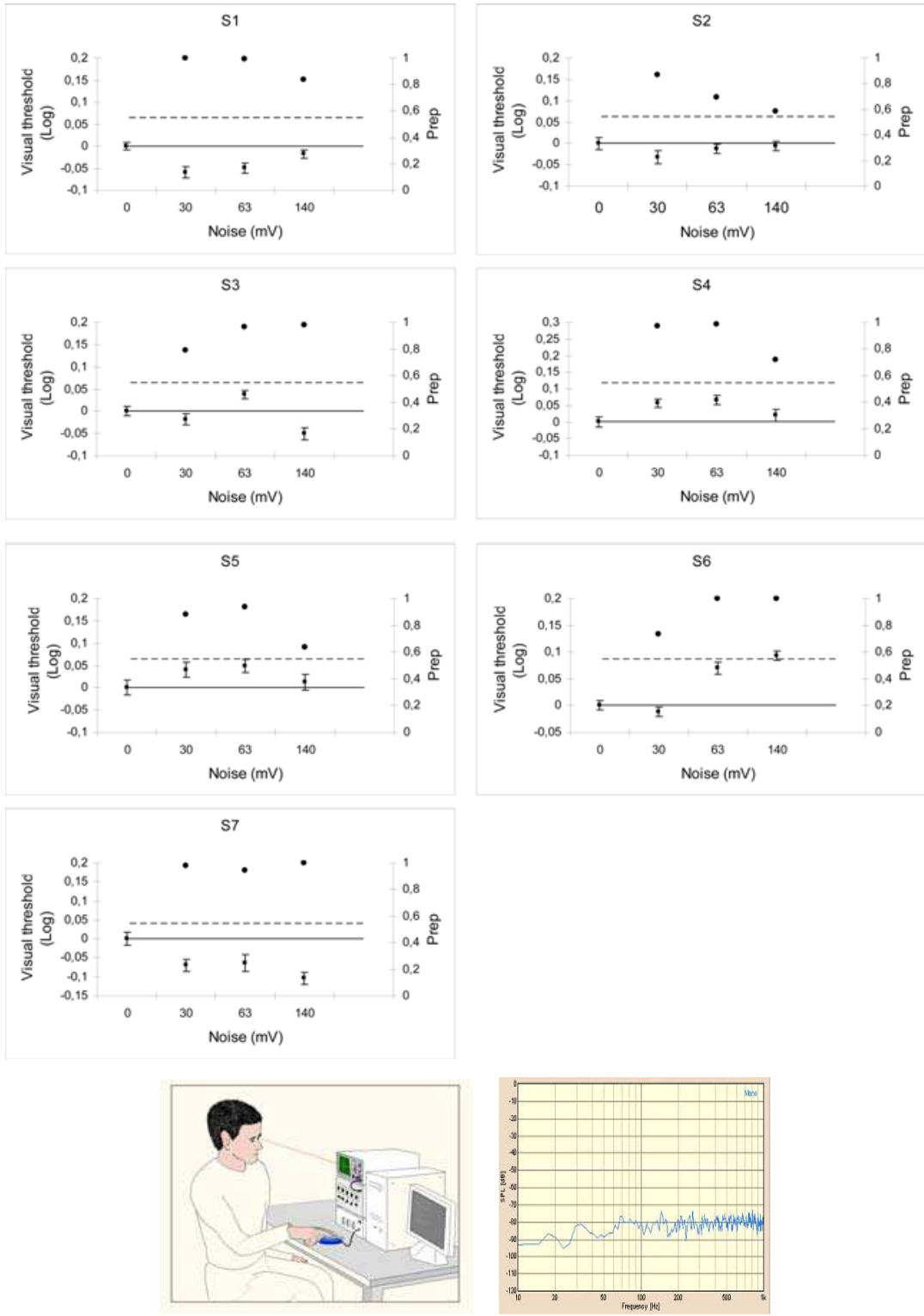

Fig. 5. Interactions between tactile noise and first order visual signals. Normalized visual threshold changes with the noise level in seven subjects for luminance modulated (first order) stimuli. In all the graphs the no-noise condition is taken as baseline; the black dots indicate the probability to replicate the same result (right y-axis) and the broken line represents the $50 \%$ chance level. Error bars correspond to one standard error. (Last row) shows an example on how the experiments were done and the effective tactile noise Fourier spectral density. 
The normalized PSD was used to calculate the integral signal-to-noise ratio (integral SNR), defined as follows:

$$
\text { Integral.SNR }=\int_{-\infty}^{\infty} \Psi_{N}(\omega) \Theta d \omega / \int_{-\infty}^{\infty} \Theta d \omega
$$

where $\Theta$ is a step function that equals zero when $\Psi_{\underline{N}}(\omega)<1$ and equals one otherwise. On each trial, we obtained two paired measurements: the EMG for a tactile stimulus at a subthreshold level with a fixed amplitude (1.5\% below threshold) and the EMG for a tactile stimulus that was presented concurrently with a stimulus in another modality, depending on the experiment. Every EMG measurement lasted $30 \mathrm{~s}$, and the order of the paired measurements within each trial was randomized to ensure that the observed effects were not simply due to a generalized modulation in attention or arousal

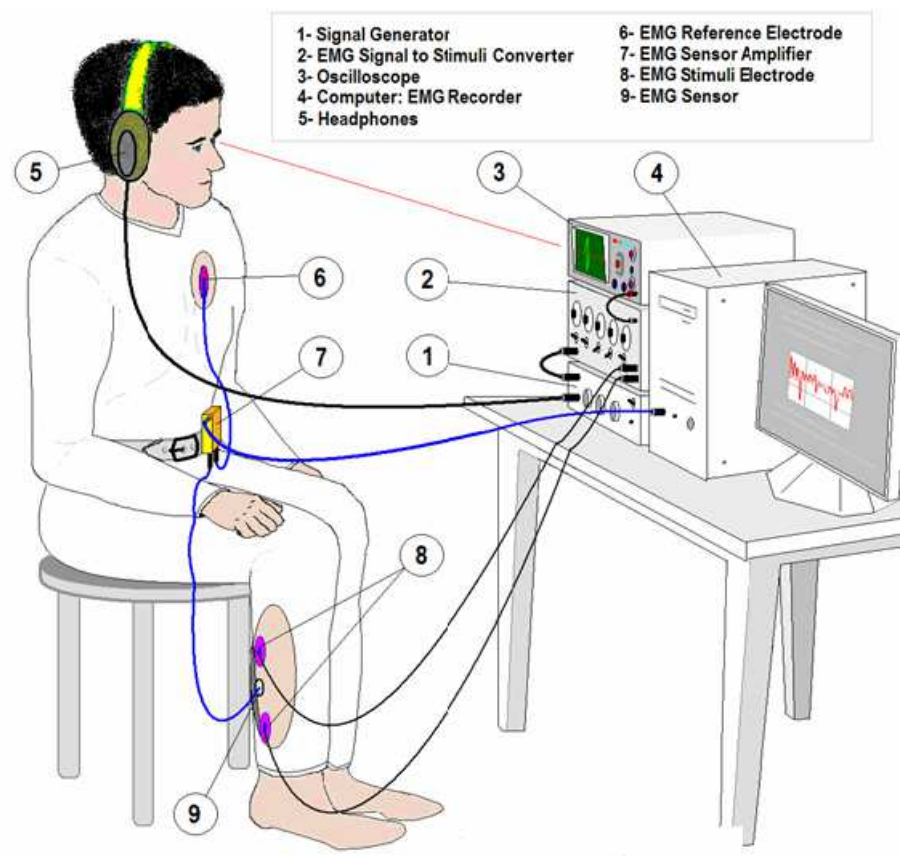

Fig. 6. Experimental lay-out for all the procedures related to deterministic signals described in the text, including the nine components of the experiment set-up.

\section{Excitatory: Tactile - Deterministic- Sub threshold $\quad$ E:T-D-ST Facilitating: Auditory - Deterministic - Supra threshold $\quad$ F: A-D- SST}

The auditory stimuli were presented binaurally by means of a pair of high-precision headphones. We evaluated first the subjects' hearing from $250 \mathrm{~Hz}$ to $8 \mathrm{kHz}$ using an audiometer; these evaluations were conducted in a $6-\mathrm{ft}$ by $10-\mathrm{ft}$ double-wall audiometric sound suit that met the American National Standards Institute (Standard 3.1-1991) for permissible ambient noise levels (in one-third-octave bands) for testing in free-field conditions with headphones. During the experimental trials, all subjects were seated and 
were asked to listen to the sound in the headphones and report when they first felt a tactile sensation. Once the subjects reported a change in tactile sensation, the EMG measurements started. The electrical amplitude signal was set to a subtreshold level (1.5\% below threshold) and the auditory signal had a fixed amplitude of $9 \mathrm{mV}$ (peak voltage). Figure 7a shows an example of the normalized power spectral density PSD. The enhancement ranges between $3 \%$ and $9 \%$ for all the subjects (fig. $7 b$ ).
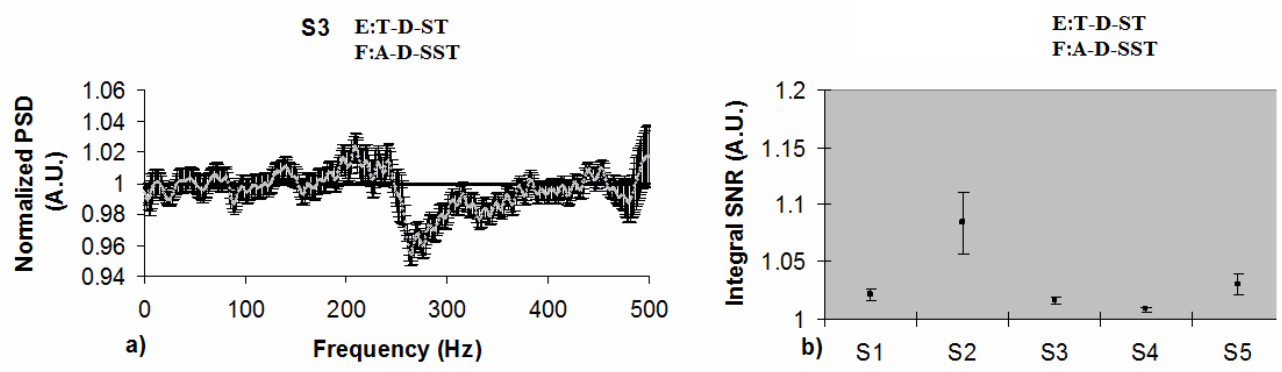

Fig. 7. a) An example of the normalized PSD of subject S3 for tactile-auditory interactions of deterministic signals. The grey line represents the mean and the black bars indicate one standard error. b) The graph in the second column shows the integral SNR for five subjects.

\section{Excitatory: Tactile - Deterministic- Sub threshold E:T-D-ST \\ Facilitating: Visual - Deterministic- Supra threshold F:V-D- SST}

Second, we investigated how tactile perception and the corresponding EMG activity were affected when the amplitude of the tactile stimulus was subthreshold $(1.5 \%$ below threshold) and a suprathreshold visual stimulus was presented concurrently. The biphasic visual signal (Component 3) was displayed on an oscilloscope (Kikusui COS6100) and looked like a dot expanding to a line, first up and then down. All subjects were seated $45 \mathrm{~cm}$ from the oscilloscope screen and were asked to look at the screen and report when they first felt a tactile sensation. Once the subjects reported a change in tactile sensation, the EMG measurements started. The visual stimulus augmented tactile perception and the corresponding EMG activity. When we introduced the visual stimulus, the EMG activity increased correspondingly, primarily in frequencies between 290 and $380 \mathrm{~Hz}$ (Fig. 8a displays the EMG results from 1 subject). Figure $8 \mathrm{~b}$ shows the integral SNR for all subjects, which ranged from approximately 1.03 (increase of 3\% relative to baseline) to 1.1 (increase of $10 \%)$.

Can we explain the results of the last two experiments in terms of MI? The first condition for MI, temporal synchronicity, was satisfied in our experiments, because the two stimuli were presented at the same time. However, because the visual and auditory stimuli were suprathreshold and the tactile stimuli were subthreshold, the inverse-effectiveness rule seems not to be applicable to this case (greatest multisensory-mediated effects are generally seen when the individual stimuli are both weak in eliciting a response on their own). Therefore, we predicted (a) that visual or auditory noise also enhances tactile sensations, and (b) that there is a particular intermediate level of visual or auditory stimulation at which tactile-visual or tactile-auditory MI is optimally enhanced. We tested these predictions in the next two experiments by using auditory stimuli only. 

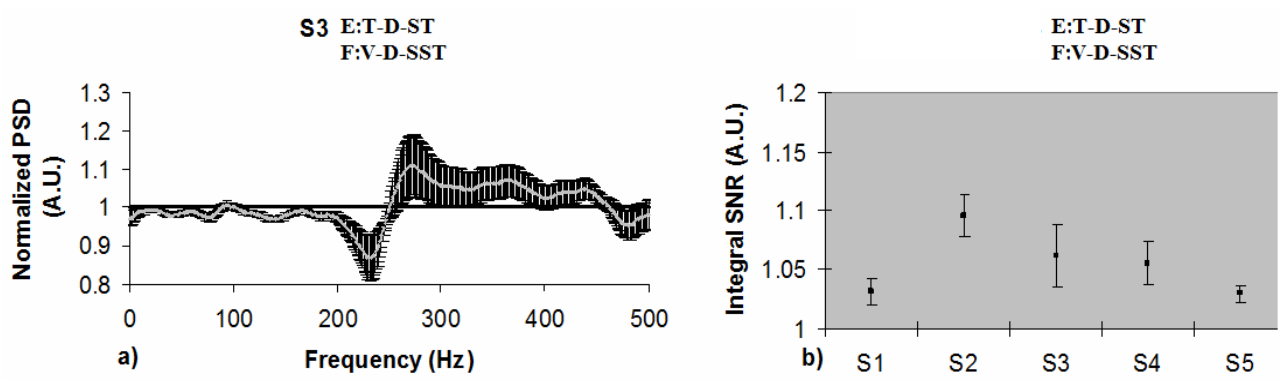

Fig. 8. a) An example of the normalized PSD of subject S3 for tactile-visual interactions of deterministic signals. The grey line represents the mean and the black bars indicate one standard error. b) The graph in the second column shows the integral SNR (left y-axis) for all subjects.

First, we tested tactile-auditory interaction using auditory noise instead of a deterministic auditory signal. In this experiment, we tested only the 3 subjects whose results for tactileauditory interactions were similar. In this experiment, a subthreshold tactile stimulus $(1.5 \%$ below threshold) was presented concurrently with a clearly audible noise stimulus (rather than the deterministic auditory stimulus). The amplitude of the white-noise signal was fixed at a value of $9 \mathrm{mV}$ (peak voltage) and it has an effective acoustic noise spectrum (ENS).We estimate that the ENS upper bound is around $15 \mathrm{kHz}$.

Figure 9a indicates that the auditory noise enhanced tactile sensations because, on average, the EMG signal increased when the auditory noise was present. In addition, the integral SNR (see Fig. 9b) ranged from 1.05 (increase of $5 \%$ relative to baseline) to 1.10 (increase of $10 \%$; similar to the range of tactile-visual SNRs), indicating that the energy transfer of the auditory noise was bigger than the energy transfer of the deterministic auditory signal. These differences in energy transfer could have been due to the fact that the frequency content was larger in the auditory noise signal than in the auditory deterministic signal. This would imply that the frequency content, and not just the energy content, is important in inducing transitions in tactile perception.
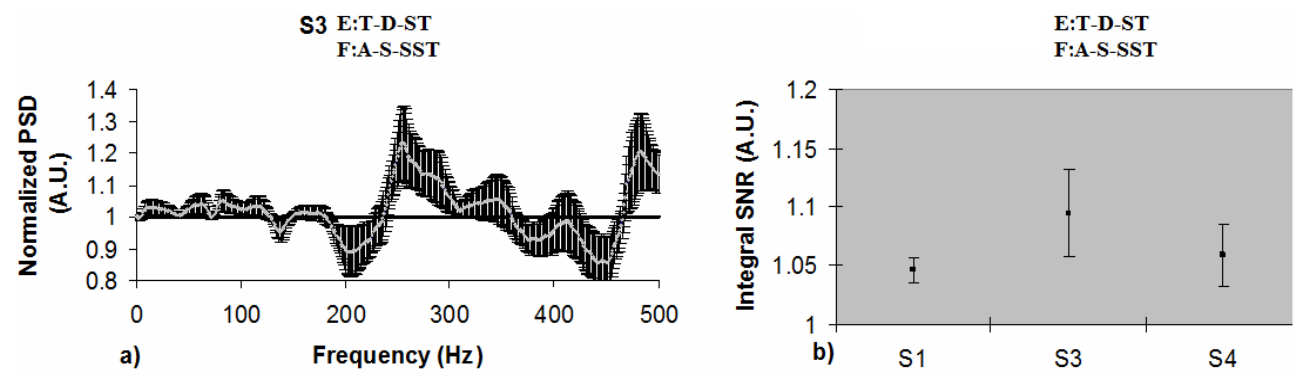

Fig. 9. a) An example of the normalized PSD of subject S3 for tactile interactions with auditory noise. The grey line represents the mean and the black bars indicate one standard error. b) The graph in the second column shows the integral SNR (left y-axis) for three subjects. 
Finally, in the last experiment, we tested tactile-auditory interaction using deterministic auditory signals with different amplitudes and measured EMG activation in 1 subject (S4). A different amplitude of the auditory signal was tested at each session. The six amplitudes were $0,8,12,20,30$, and $300 \mathrm{mV}$ (peak voltage) at the amplifier exit. To show the invertedU-shaped function, we chose the upper limit to be $300 \mathrm{mV}$.We kept the intensity of the continuous auditory stimulus constant within each session and varied the intensity (in random order) between sessions. The order of the paired measurements was randomized within each trial (as in the previous experiments), and the order of the sessions was also randomized; this randomization ensured that the observed effects were not simply due to a modulation in attention or arousal. Figure 10 demonstrates that as we increased the amplitude of the auditory stimulus, EMG activity increased, reached a maximum, and then decreased (inverted- U-shaped function). This implies that there is indeed a particular intermediate level of auditory stimulation at which tactile auditory MI is optimally enhanced. Surprisingly, the same pattern of results shown in Figure 10 has been demonstrated in systems that show SR, deterministic resonance, or both [32].

\section{S4 E:T-D-ST \\ F:A-D-SST}

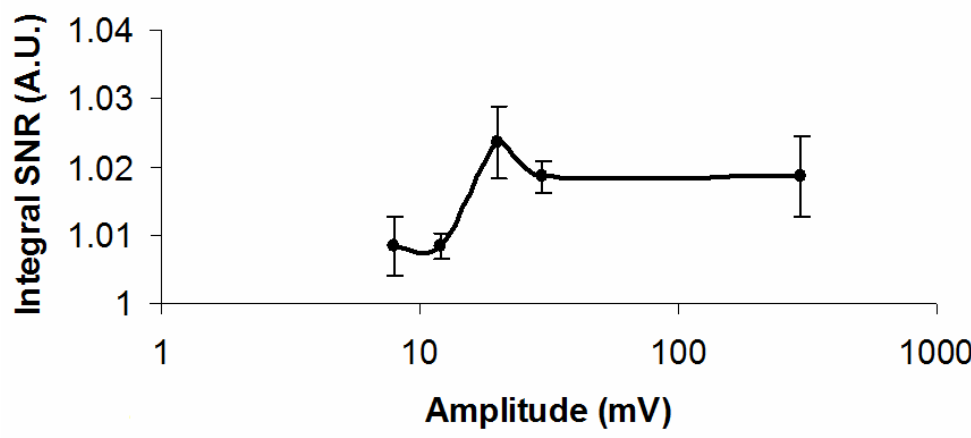

Fig. 10. Results for tactile-auditory interactions with deterministic auditory signals at different amplitudes. The integral signal-to noise ratio (SNR) of 1 subject (S4) is shown for the full frequency range of the electromyographic signal, from 0 through $500 \mathrm{~Hz}$

\section{The Fulcrum principle}

SR was shown to be capable of improving sensitivity for sub threshold excitatory stimuli. At the beginning SR was thought as a local peripheral effect. But evidence has confirmed the ubiquitous influence of the facilitating stimulus by triggering a mechanism that involves the cortex acting upon the peripheral sensory activity. And this mechanism was shown for both, stochastic and deterministic supra threshold facilitating signals as part of a general principle for the stimuli interactions that could explain all the paradigms.

So, experiments 2 to 7 permitted to introduce a General Dynamics Model that involves the entire MI threshold enhancement. This non-linear model that handles deterministic or stochastic facilitating signals has shown be useful for explaining all the paradigms and we therefore call it: The FULCRUM Principle. A fulcrum is one that supplies capability for action and we believe that this best describes the fundamental principle at work in these 
multisensory interactions. The principle can be summarized as follows: a subthreshold excitatory signal (entering in one sense) that is synchronous with a facilitation signal (entering in a different sense) can be increased (up to a resonant-like level) and then decreased by the energy and frequency content of the facilitating signal. As a result the sensation of the signal changes according with the excitatory signal strength. In this context, the sensitivity transitions represent the change from spontaneous activity to a firing activity in multisensory neurons. Initially the energy of their activity (supplied by the weak signals) is not enough to be detected but when the facilitating signal enters the brain, it generates a general activation among multisensory neurons, modifying their original activity. In our opinion, the result is an integrated activation that promotes sensitivity transitions and the signals are then perceived. In other words, the activity created by the interaction of the excitatory signal (for example tactile) and the facilitating signal (auditory noise) at some specific energy, produces the capability for a central detection of an otherwise weak signal.

\section{Mathematical model for the Fulcrum}

We can simulate neurons as natural devices with dynamics that consist of random lowamplitude motions (spontaneous neuronal activity) from which escapes occur at certain intervals [32]. The escapes are referred to as firings, and are associated with high amplitude bursts (spikes). We begin by proposing a similar bistable model for the response of neurons as in [32]

$$
\ddot{x}=-V^{\prime}(x)+\varepsilon\left[\gamma \operatorname{Cos}\left(\omega_{0} t\right)+\sigma G(t)-\beta \dot{x}\right],
$$

Where $x$ represents the neurons' amplitude activity, $\dot{x}$ is the neurons' amplitude activity velocity (how their activity changes with time), $V(x)$ is a double-well potential defined by a polynomial, $\varepsilon$ is a perturbation parameter that may have a stepwise variation over $x$. $\operatorname{Cos}\left(\omega_{0} t\right)$ represents the excitatory weak signal, $G(t)$ is the facilitating signal and it can be a nearly white noise process or a deterministic one, $\gamma, \sigma$ and $\beta$ are adjustable parameters. The quantities between brackets represent excitatory, facilitating energy, and energy losses. Equation (1) can achieve simulations of neuronal time histories (with the appropriate parameter values) and it has solution with the qualitative features observed in the experiments described earlier. To achieve good neuronal time history simulations, the potential $V(x)$ must be asymmetric, which is deeper for $x>0$ than for $x \leq 0$ as shown in figure 11 (left column, top row).

\section{Neuronal firing necessary condition}

Associated with an unperturbed system $(\varepsilon=0$ for all $x)$ are the homoclinic orbits $\Gamma^{+}$and $\Gamma^{-}$shown in figure 11 (left column, middle row). In order for the escapes to take place we require that the maximum total energy produced during the motion over an entire homoclinic loop will be bigger than zero. Suppose the motion takes place on the unperturbed system's homoclinic orbit. If the motion occurs over a small distance $\delta x_{h}(h$ designates coordinates of the homoclinic orbit), then the maximum total energy is given by :

$$
E_{\text {tot }}=E_{\text {loss }}+E_{\text {exc }}=-\varepsilon \beta \int_{-\infty}^{\infty} \dot{x}_{h}^{2} d t+\varepsilon \int_{-\infty}^{\infty}\left\{\gamma \operatorname{Cos}\left[\omega_{0}(t)\right]+\sigma G(t)\right\} \dot{x}_{h} d t
$$


The condition $\max \left(E_{t o t}\right)>0$ implies that the maximum of the second term between braces in equation (2) is larger than the first term. This implies that the energy of the system can drive the motion over the potential barrier and out of a potential well.

\section{Fulcrum neuron firing condition}

It is possible to show that the necessary condition for the Fulcrum to occurs [5], for the stochastic process $G(t)$, is

$$
-4 \beta \sqrt{\alpha} / 3+\gamma S\left(\omega_{0}\right)+\sigma \sum_{k=1}^{N} a_{k} S\left(\omega_{k}\right)>0
$$

where the constants $a_{k}$ are related to the Fourier one-side spectral density. For a second harmonic signal $\sigma \operatorname{Cos}\left(\omega_{1} t\right)$ instead of white noise the conditions writes:

$$
-4 \beta \sqrt{\alpha} / 3+\gamma S\left(\omega_{0}\right)+\sigma S\left(\omega_{1}\right)>0 \text {. }
$$

where $S\left(\omega_{j}\right)=(2 / \alpha)^{1 / 2} \pi \omega_{j} \operatorname{sech}\left\{\pi \omega_{j} / 2 \sqrt{\alpha}\right\}$ is known as the Melnikov scale factor. It is clear that if we want to optimize the energy transfer from the stochastic process $G(t)$ or deterministic process $\sigma \operatorname{Cos}\left(\omega_{1} t\right)$ then the spectral density of $G(t)$ needs to contain frequencies around the Melnikov scale factor maximum and the frequency $\omega_{1}$ from the signal $\sigma \operatorname{Cos}\left(\omega_{1} t\right)$ must be centered at the Melnikov scale factor $S(\omega)$ peak as well. The central column in figure 11 shows the neurons spectrum amplitude as a function the noise intensity $\sigma$. As it is expected for low noise intensities the energy transfer from the noise to the signal is not enough to achieve the synchronization and as a result the spontaneous activity dominates and no firings occur. However as the noise intensity increases firings also increase up to a maximum peak, where the mean escape rate approximately equals the signal frequency. Beyond this point, random firings can occur at different frequencies meaning that the synchronized energy transfer from the noise to the signal is destroyed and the signal is embedded in the spontaneous activity. The insert (center column, middle row) shows the well-known SR inverse u-shape function and its maximum peak. Right column in figure 11, shows neuron firing histograms with their correspondent time histories. It is clear from Equations (3) and (4) that if we increase the energy losses we have to increase accordingly the excitatory energy to fulfill the fulcrum neuron firing condition always. This means that the energy transfer is always fixed no matter how long is the neuronal network.

\section{Consequences of the Fulcrum principle}

The first consequence is that signals in the peripheral nervous system can be modulated by crossmodal interaction at the central level as we have seen clearly from examples 6 and 7 . What normally could be considered to be a simple, peripheral, and reflexive muscular reaction to a directly applied stimulus turns out be a multimodal function. No sense, even the most peripheral, works on its own [33]. Indeed, the energy and frequency content of the facilitating signal induces the transition in perception of the excitatory signal. However, we are not proposing that the sensory activity is only peripheral. Initially, the energy level of 

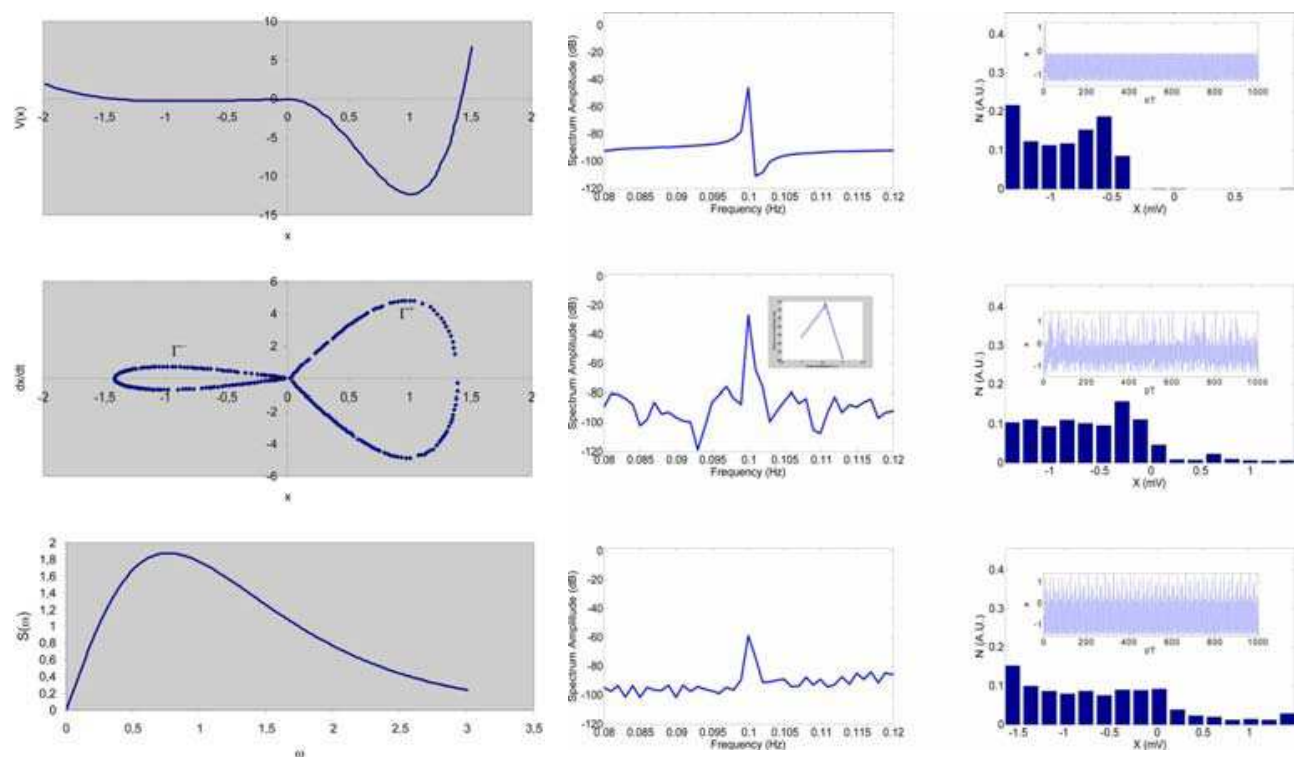

Fig. 11. Theoretical model for the fulcrum. (Left column, top row) Potential V(x); (Left column, middle row) Phase plane diagram showing homoclinic orbits; (left column, bottom row) Melnikov scale factor; (Center column) shows the neurons' spectrum amplitude as a function of the noise intensity. The insert (Center column, middle row) shows the wellknown SR inverted u-shape function. Right column shows neuronal firing histograms with their corresponding time histories. $\mathrm{T}$ is the signal period and $\mathrm{N}$ means the probability to have certain neuronal activity levels.

the peripheral activity is not high enough to be detected by the central system; therefore, there is no interaction between central and peripheral systems at that time. When the facilitation signal enters the central system, it generates an activation that goes all the way back and modifies the original peripheral activity. The result is an activation that promotes resonance-like behavior, increasing the peripheral signal up to a level where it is perceived by the central system. This means that once the peripheral signal is perceived, the integration is represented not only at a central level, but also at a peripheral level. At some energy level of the facilitating stimulus, the peripheral activity reaches a maximum, and peripheral activity begins to decrease if the energy is increased further (see Fig. 10). Because the increase in peripheral activity comes from the way the brain processes the energy and frequency content of the facilitation signal in each individual, the nature of the signals (deterministic or stochastic) involved in the interactions is not important. If the facilitation signal has the right energy and frequency content, the phenomenon will occur. That is why deterministic signals (visual or auditory) and a stochastic signal (auditory noise) demonstrated the same effects in our experiments.

A second consequence is that these MI interactions do not follow the inverse-effectiveness rule, but are consistent with the fact that tactile, visual, and proprioceptive detection, and audiovisual comprehension of spoken words are substantially improved at an intermediate level of auditory noise [5,23,34-35]. 
A third consequence is the possibility to explain properties of the Yerkes-Dodson law dynamics under certain conditions. The Yerkes-Dodson law is an empirical relationship between performance and arousal, and it was developed in 1908 [26]. This law establishes that performance increases with physiological or mental arousal, but only up to a point. When the arousals become too high, performance decreases. The law is illustrated graphically as an inverted U-shaped curve which increases and then decreases with higher levels of arousal. Nonetheless, it is known that for simple or well learned tasks the relationship can be considered linear with improvements in performance as arousal increases. For difficult tasks however, the relationship between arousal and performance becomes inverse, with declines in performance as arousal increases. There has been research indicating that the correlation suggested by Yerkes and Dodson exists but a causal explanation of the correlation has not yet been successfully established [36]. Since we are using auditory noise, one might argue that $70 \mathrm{dBSPL}$ (clearly audible) could be judged annoying by some people (although previous crosmodal SR claims have shown that this is the effective range [23]). Indeed sound annoyance is a complex thing and no single level can be pointed to as a threshold for it, there are reports of high levels of annoyance for very soft sounds indeed (e.g. 35dBA sound of a toilet flushing from an apartment above) [37]. Annoyance is defined by the context, and $70 \mathrm{~dB}$ SPL white noise for a normal hearing person could easily be construed as annoying under some conditions, for example if it were perceived to affect performance in an experiment where the participant wanted to do well. Indeed, subjects were exposed to white noises from 60-95 dB SPL during the experiment, so the noise could have been construed as interfering and annoying at all of the levels used, and could have caused arousal optimal for the task at around $70 \mathrm{~dB}$. From these arguments one could possibly advance the hypothesis that the crossmodal effects are due to arousal. Arousal is a physiological and psychological state of being awake and represents physiological readiness for activity. Readiness or preparedness is the state of having been made ready or prepared for use or action. We argue that this classic definition of arousal cannot account for the crossmodal facilitation results presented here and elsewhere [23,5- 6] for several reasons. First our experimental conditions were all randomized and our subjects naïve, which would reduce the possibility of being specifically prepared for one condition or another. Second, we have shown that we can obtain similar dynamics with deterministic signals experimentally [6] and via modeling as well [5]. Given that the deterministic facilitation signals were simultaneously paired with the detection signal (no anticipation) we can also argue that the classic definition of arousal from noise would fall short at explaining these dynamics. Further, from the model we have developed it is clear that it is not the stochastic process that defines the noise (its uncontrollable nature) that makes the synchronization-like phenomenon occur. Instead it is the energy and frequency that are contained in the noise (or a harmonic signal) and the interaction between the excitatory and facilitation signals that makes the phenomenon possible and allows the subjects to improve perception. Another argument can be made against a simple arousal interpretation of our experiments. We found that the crossmodal SR effect was similar between luminance versus contrast-defined stimuli. It is well known that such stimuli require different processing levels where the contrast-defined stimuli are more complex to process $[27,28]$ and are differentially affected by other factors such as attention, fatigue and learning. We would therefore have expected a greater and different effect of arousal on the contrast defined stimuli but we did not find this. Rather we found very similar results and this would be difficult to account with a simple arousal explanation. Nonetheless the fact that 
the subject's perception is enhanced by SR mechanisms might change the subjects' behavior if we would ask them to do a second task in parallel with the detection task such as in behavioral SR [38]. This implies that known behavioral effects induced by noise or other deterministic signals may have their origin at a lower level. We therefore propose that the fulcrum possibly explains properties of the Yerkes-Dodson law dynamics under certain conditions.

From unimodal SR studies it can be inferred that $70 \mathrm{dBSPL}$ is much louder than the noise required for auditory SR [39-40]. This may make the SR label we have used here problematic. However the auditory unimodal SR works in a simpler architecture than the crossmodal SR [5], where larger neuronal networks are necessarily involved between modalities. Since the crossmodal architecture is vaster, and complex, one would expect more energy losses in such network and according with the model we have developed it is possible to have synchronized neuronal firings with these conditions. The aforementioned studies have shown that auditory unimodal SR happens between $5 \mathrm{~dB}$ [39] and 3-5 dB [40] below a point defined as noise threshold [40]. The noise threshold is the point where the noise hinders the signal detection and the sensitivity worsens to levels above threshold (the crossing point in the inverse u-shape curve). If we use this level as our reference instead of the SPL absolute scale (we will call this level the noise ceiling level that defines a ceiling decibel $\mathrm{dBc}$ ) then we found that crossmodal SR threshold minima occur approximately in the same experimental range as the ones mentioned above. We found that for visual experiments the minima are localized at $-6 \pm 1 \mathrm{dBc}$ (first order) and $-5 \pm 1 \mathrm{dBc}$ (second order). In the proprioception experiments the minima occurs around $-6 \pm 1 \mathrm{dBc}$ and for tactile experiments at $-8 \pm 1 \mathrm{dBc}$ and for the experiment 6 with one subject (figure 10) $-4 \mathrm{dBc}$. These results underscore the very important fact that independently of the unimodal or crossmodal interaction the energy transfer from signal plus noise is approximately fixed, which is the fourth consequence of the fulcrum. Note that for measuring the noise ceiling level we have used a similar approach than the one presented in [40].

Implications for autism: The fifth consequence is related to a better understanding of disorders such as autism, in which altered sensory processing often occurs that causes perceptual dysfunction, it causes problems with one or more sensory channels from the world to the brain. In a very general classification given in [41] the sensory channels are abnormal in one of the followings ways: Hyper: the sensory channel is too open and, as a result, too much stimulation gets in for the brain to be handled comfortably. Hypo: the sensory channel is not open enough and, as a result, too little of the stimulation gets in and the brain is deprived. White noise: the sensory channel creates its own stimulus because of faulty operation and, as a result, the message from the outside world is garbled or, in extreme cases, is overcome by the noise in the system. The broad autism classification can be qualitatively understood by using the neuron firing condition:

$$
-4 \beta \sqrt{\alpha} / 3+\gamma S\left(\omega_{0}\right)+\sigma \sum_{k=1}^{N} a_{k} S\left(\omega_{k}\right)>0
$$

Let us assume in this case that the stochastic energy is due to the internal noise and the excitatory signal is deterministic. Therefore the Hyper type can be described by: small values of parameters $\beta$ and $\alpha$ that make the energy losses small. This represents an internal noise energy level close to the energy losses level and a Melnikov scale factor that is very narrow (this is because $\alpha$ is small). With these conditions a very weak excitatory signal elicits neuronal firing and because of the Melnikov narrowness factor, the optimal condition 
for the firings is easily achieved (but only for a small frequency bandwidth). Note that if the frequency content of the excitatory signal is outside of this bandwidth then the classification will change to Hypo. Hypo condition: High values of parameters $\beta$ and $\alpha$ that make the energy losses high. Therefore, the internal noise energy is lower than the energy losses. This represents a Melnikov scale factor that is very broad (this is because $\alpha$ is high). With these conditions a very strong excitatory signal is needed to elicit neuronal firing and, because the Melnikov factor broadens, more frequencies can induce the optimal condition for the firings with less selectively than the Hyper type. White noise condition: the stochastic energy is higher than both the energy losses and the excitatory signal combined. Then neuronal firings occur but they are mainly driven by the internal stochastic process. Note that nothing precludes that sensory channels present one or more classifications. That is, a person might be hyper or hypo for the different stimuli because optimal neuron firings depending on the frequency and energy content of the involved signals.

\section{More experimental evidence for the Fulcrum Principle}

The consequences of spinal cord injury or Parkinson's disease are not just a break in communication between neurons; a cascade of events occur that promote further neuronal degeneration, cell death and motor dysfunctioning [42]. Locomotion training is a very effective tool in neuronal degeneration rehabilitation. Besides regular locomotion exercises (or similar strategies) are associated with neuroprotective effects in different brain areas. Nevertheless, numerous patients are unable to do locomotion therapy and therefore the possibility of rehabilitation is reduced. Haas [43] has bypassed this problem by using vibratory stimulations, leading to reflex responses similar to reflex elicitations during human locomotion. He found that stochastic mechanical stimulations might be a useful method to counteract neuronal degeneration and to promote regenerative processes. His patients either stood up or sat down in a special chair and both legs were connected with two independently oscillating platforms. The platforms could oscillate with a mean frequency of $6 \mathrm{~Hz}$ and superimposed by random and stochastic influences which facilitate neuronal threshold crossing and enhance neuromuscular activity. Patients with Parkinson's disease and spinal-cord-injury patients that were stimulated regularly lead to significantly improved postural control and locomotion abilities. Interestingly, treated Parkinson's disease patients also showed reduced symptoms (tremor, rigidity) in the upper extremities. As improvements in manual coordination (for instance writing performance) were confirmed in further standardized experimental setting, it seemed unlikely that this vibratory stimulation affected only the muscle or exclusively the peripheral nervous system. That is, if only the lower limbs were excited, how could we explain improved writing performance?

The startle reflex is the response of brain and body to an unexpected stimulus, such as a loud noise, a flash of light, or a sudden movement near the face. The reaction includes physical movement away from the stimulus, a contraction of the muscles of the arms and legs, and often blinking. The startle reflex provides a unique tool for the investigation of sensorimotor gating and information processing. Neuner et al. presented the first MR study using a single trial approach with simultaneous acquired EMG and fMRI data on the human startle response [44]. The startle reflex was recorded from the right orbicularis oculi muscle. Electrodes for recording electromyographic activity of this muscle were fixed below the eye in midline and the outer canthus. The air puffs were delivery to the region below the left clavicula. They investigated the neural correlates for isolated air puff startle pulses (PA), 
prepulse-pulse inhibition (PPI), and prepulse facilitation (PPF), via air puffs onto the skin. PPI is a neurological phenomenon in which a weaker prepulse inhibits the human reaction to a subsequent strong startling stimulus. PPI is present in a vast number of species (from mice to human) and usually is measured through muscular reactions, which are normally diminished as a result of the nervous inhibition. The opposite reaction is known as prepulse facilitation (PPF). A common core network engaged by all three conditions (PA, PPI, and PPF) was identified. The network consists of bilateral primary and secondary somatosensory cortices, right insula, right thalamus, right temporal pole, middle cingulate cortex, and cerebellum. The cerebellar vermis exhibits distinct activation patterns between the startle modifications. It is differentially activated with the highest amplitude for PPF, a lower activation for PA, and lowest for PPI. The orbital frontal cortex exhibits a differential activation pattern, not for the type of startle response but for the amplitude modification. For pulse alone it is close to zero; for PPI it is activated. This is in contrast to PPF where it shows deactivation. In addition, the thalamus, the cerebellum, and the anterior cingulate cortex add to the modulation of the startle reflex. In summary, this research shows that peripheral activation, through somatosensory stimulation and measured with EMG techniques, correlates with central activation measured with fMRI techniques.

Fine-motor performance of the hand is more than important in our life and work. However, some injuries might damage the hand fine-motor performance. Previous studies show that fine-motor performance of the hand might be improved according to the mechanism of coactivation [45]. Lei Ai et al. tested if fine-motor performance could be enhanced by the presence of auditory noise. They used a pegboard which has 50 holes arranged in four rows and 50 metal pins placed in a container. In every trial subjects were asked to pick these 50 pins with their right hand, one by one, from the container and insert them into 50 holes on the peg-board. Every hole is inserted by one pin. If one pin is dropped during the transfer, they were instructed to pick the next pin from the container to insert the hole that they just failed. The dependent variable was the length of time required for completing the process of inserting all the pins. The less the time the more dexterous the hand is. The result was a Ushape function of the intensity of different levels of auditory noise showing that optimal auditory noise can largely improve the fine-motor performance.

Noise is typically conceived of as being detrimental to cognitive performance. However, a certain amount of noise can benefit performance. Soderlund et al. investigated cognitive performance in noisy environments in relation to a neurocomputational model of attention deficit hyperactivity disorder (ADHD) and dopamine [46]. They hypothesized that dopamine levels modulate how much noise is required for optimal cognitive performance. They experimentally examined how ADHD and control children responded to different encoding conditions, providing different levels of environmental stimulation. Participants carried out a high memory performance task and a low memory task. These tasks were done in the presence, or absence, of auditory white noise. They found that noise exerted a positive effect on cognitive performance for the ADHD group and deteriorated performance for the control group, indicating that ADHD subjects need more noise than controls for optimal cognitive performance.

\section{Final remarks}

The Fulcrum principle describes a ubiquitous process in humans related in how our peripheral and central systems use energy and frequency content of external and internal 
signals to modulate our perception of reality. We have seen that stochastic or deterministic sounds of one modality can facilitate perception of stimuli in another modality and underscore that they share the same dynamics. This and the fact that the energy transfer necessary for neuronal firings to occur is approximately constant for all the interactions presented here, implies that the central system also can modulate the peripheral system. Consequently, no single sense works alone. These interactions may be the basis for explaining certain aspects of arousal dynamics related with the Yerkes-Dawson law. At the same time they challenge us because they seemingly do not follow the inverse effectiveness law from the classic multisensory integration theory. We propose that the fulcrum principle also gives us a theoretical framework for a better understanding of autism an ADHD conditions. Finally, these results have obvious implications in developing methods for enhancing human performance in easy non-invasive ways. One possible application is with Parkinson disease as we have seen stochastic vibrations applied to lower limbs not only enhanced mobility and decreased tremors in the same anatomical part where the vibration was applied but on the upper limbs as well. We know from our results that stochastic sound should also work. Spinal cord, motor system, memory, ADHD and Alzheimer diseases may be treated with the same acoustic therapy that would be beneficial for everyone and in particular to the elderly. As we age, we depend more and more on multisensory perception and it has been recently suggested that, despite the decline in sensory processing that accompanies aging, the use of multiple sensory channels may represent an effective compensatory strategy to overcome uni-sensory deficits [47]. In summary, in the presence of any one sensory deficit or any neurobiological alteration the Fulcrum principle takes on a new and important meaning.

\section{References}

[1] Georg Von Békésy (1967), Sensory inhibition, Princeton University Press, Princeton NJ,

[2] Georg Von Békésy (1957), Sensations on the skin similar to directional hearing, beats, and harmonics of the ear, Journal of the Acoustical Society of America, 29: 489-501.

[3] Georg Von Békésy (1964), Rhythmical variations accompanying gustatory stimulation observed by means of localization phenomena, Journal of General Physiology, 47:809-825.

[4] Georg Von Békésy (1964), Olfactory analogue to directional hearing, Journal of Applied Physiology, 19: 369-373.

[5] Lugo E, Doti R, Faubert J (2008) Ubiquitous Crossmodal Stochastic Resonance in Humans: Auditory Noise Facilitates Tactile, Visual and Proprioceptive Sensations. PLoS ONE 3(8): e2860. doi:10.1371/journal.pone.0002860

[6] The final, definitive version of this paper has been published in Psychological Science, 19:989-997, 2008 by SAGE Publications Ltd./SAGE Publications, Inc., All rights reserved. (C)

[7] Lugo J E, Doti R and Faubert J (2010), Effective tactile noise can decrease luminance modulated thresholds, Journal of Vision 10 doi:10.1167/10.7.857.

[8] Schwartz M (1977), Information, Transmission, Modulation, and Noise, Mc Graw-Hill book Co., New York, N.Y.

[9] Athanasios Papoulis (1959), SIGNAL ANALYSIS, Page 12 Chapter 1-2: Analog Signals and Systems, Mc Graw-Hill book Co., New York, N.Y. 
[10] Athanasios Papoulis (1977), SIGNAL ANALYSIS, Page 184 Chapter 6-1: Properties of Band-limited Functions, Mc Graw-Hill book Co., New York, N.Y.,.

[11] Athanasios Papoulis (1977), SIGNAL ANALYSIS, Page 300, Chapter 9: Stochastic Processes, Mc Graw-Hill book Co., New York, N.Y.

[12] Stein B E, \& Meredith M A (1993), The merging of the senses, Cambridge: MA MIT Press..

[13] Moss F, Ward L, Sannita W (2004) Stochastic resonance and sensory information processing:a tutorial and review of application. Clinical Neurophysiology 115:267281.

[14] Benzi R, Stuera A, Vulpiani A (1981) J. Phys. A 14:L453.

[15] Simon A J, Libchaber A J (1992) Escape and Synchronization of a Brownian Particle. Physical Review Letters 68 (23):3375-3378.

[16] Badzey RL, Mohanty P (2005) Coherent signal amplification in bistable nanomechanical oscillators by stochastic resonance. Nature 437(7061):995-998.

[17] Ivey C, Apkarian A V, Chialvo D R (1998) Noise-Induced Tuning Curve in Mechanoreceptors. J. Neurophysiol. 79 (4):1879-1890.

[18] Simonotto E, Massimo R, Seife C, Roberts M, Twitty J, et al. (1997) Visual Perception of Stochastic Resonance. Physical Review Letters 78: 1186-1189.

[19] Collins J J, Imhoff T T, Grigg P (1997) Noise-mediated enhancements and decrements in human tactile sensation. Physical Review E 56: 923-926.

[20] Hidaka I, Nozaki D, Yamamoto Y (2000) Functional stochastic resonance in the human brain: Noise induced sensitization of baroreflex system. Physical Review Letters 85: 3740-3743.

[21] Kitajo K, Nozaki D, Ward LM, Yamamoto Y (2003) Behavioral stochastic resonance within the human brain. Physical Review Letters 90:218103.

[22] Harper D W (1979) Signal detection analysis of effect of white noise intensity on sensitivity to visual flicker. Percept. Mot. Skills 48: 791-798.

[23] Manjarrez E, Mendez I, Martinez L, Flores A, Mirasso C R (2007) Effects of auditory noise on the psychophysical detection of visual signals: Cross-modal stochastic resonance. Neuroscience letters 415: 231-236.

[24] Kitajo K, Doesburg S M, Yamanaka K, Nozaki D, Ward L M, et al. (2007) Noiseinduced large-scale phase synchronization of human-brain activity associated with behavioural stochastic resonance. Europhysics Letters 80 (4):40009.

[25] Shulgin B, Neiman A, Anishchenko (1995) Mean Switching Frequency Locking in Stochastic Bistable Systems Driven by a Periodic Force. Physical Review Letters 75(23):4157-4160.

[26] Yerkes R M, Dodson J D (1908) The relation of strength of stimulus to rapidity of habitformation. Journal of Comparative Neurology and Psychology 18: 459-482.

[27] Allard R, Faubert J (2006) Same calculation efficiency but different internal noise for luminance and contrast-modulated stimuli detection. Journal of Vision, 6(4): 322334.

[28] Allard R, Faubert J (2007) Double dissociation between first- and second-order processing. Vision Research, 47(9): 1129-1141.

[29] Priplata A A, Niemi J B, Harry J D, Lipsitz L A, Collins J J (2003) Vibrating insoles and balance control in elderly people. The Lancet 362: 1123-1124. 
[30] Priplata A., Niemi J B, Salen M, Harry J D, Lipsitz L A, et al. (2002) Noise-enhanced human balance control. Physical Review Letters 89:238101

[31] Eurich C W, Milton J G (1996) Noise-induced transitions in human postural sway. Physical Review E 54: 6681-6684.

[32] Simiu E. (2002) Chaotic Transitions in Deterministic and Stochastic Dynamical Systems. New Jersey: Princeton University Press. 178 p.

[33] Rosenblum L (2010), See What I'm Saying: The extraordinary Powers of Our Five Senses, New York London: W W Norton \& Company Inc. page 270.

[34] Ross, L.A., Saint-Amour, D., Leavitt, V.M., Javitt, D.C., \& Foxe, J.J. (2007). Do you see what I am saying? Exploring visual enhancement of speech comprehension in noisy environments. Cerebral Cortex, 17, 1147-1153.

[35]Faubert, J., Hahler, E.-M., Doti, R., \& Lugo, J.E. (2007). Auditory noise can facilitate low level visual processing. Journal of Vision,7, Abstract 866a. Available http://journalofvision.org/7/9/866/

[36] Anderson KJ, Revelle W, Lynch MJ (1989). "Caffeine, impulsivity, and memory scanning: A comparison of two explanations for the Yerkes-Dodson Effect". Motivation and Emotion 13: 1-20.

[37] Sato S, Kitamura T, Ando Y (2004) Annoyance of noise stimuli in relation to the spatial factors extracted from the interaural cross-correlation function. Journal of Sound and Vibration 277:511-521.

[38] Kitajo K, Nozaki D, Ward LM, Yamamoto Y (2003) Behavioral stochastic resonance within the human brain. Physical Review Letters 90:218103.

[39] Zeng F-G, Fu Q-J, Morse R (2000) Human hearing enhanced by noise. Brain Research 869:251-255.

[40] Ries D T (2007) The influence of noise and level upon stochastic resonance in human audition. Hearing Research 228:136-143.

[41] Delacato C H, The ultimate stranger:The autistic child, Academic Therapy Publications, Novato California, pp.77.

[42] Horner P J, Gage F H (2000), Regeneration the damaged central nervous system. Nature 407, 963-970.

[43] Haas C T (2008), Vibratory Stimulation and Stochastic Resonance Therapy: Results from studies in Parkinson's disease and spinal cord injury, Technologies of Globalization Congress Darmstadt, Germany.

[44] Neuner, I., Stöcker, T., Kellermann, T., Ermer, V., Wegener, H. P., Eickhoff, S. B., Schneider, F. and Shah, N. J. (2010), Electrophysiology meets fMRI: Neural correlates of the startle reflex assessed by simultaneous EMG-fMRI data acquisition. Human Brain Mapping, n/a. doi: 10.1002/hbm.20965.

[45] Ai L, Liu J, Liu J (2009), Using auditory noise to enhance the fine-motor of human's hand due to cross-modal stochastic resonance, J. Proceedings of the 2009 2nd International Conference on Biomedical Engineering and Informatics, BMEI 2009 , art. no. 5305070 .

[46] Söderlund G, Sikström S and Smart A (2007), Listen to the noise: noise is beneficial for cognitive performance in ADHD, Journal of Child Psychology and Psychiatry 48: 840-847.

[47] Laurienti PJ, Burdette JH, Maldjian JA, Wallace MT (2006) Enhanced multisensory integration in older adults. Neurobiology of Aging 27(8):1155-1163. 


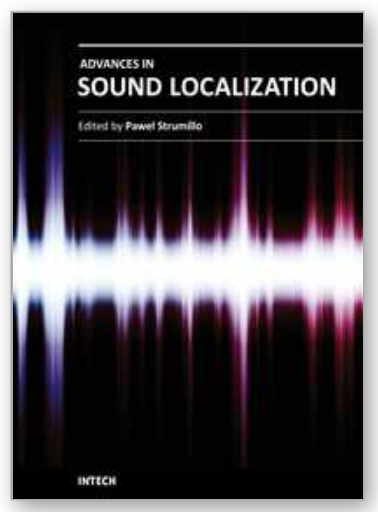

\author{
Advances in Sound Localization \\ Edited by Dr. Pawel Strumillo
}

ISBN 978-953-307-224-1

Hard cover, 590 pages

Publisher InTech

Published online 11, April, 2011

Published in print edition April, 2011

Sound source localization is an important research field that has attracted researchers' efforts from many technical and biomedical sciences. Sound source localization (SSL) is defined as the determination of the direction from a receiver, but also includes the distance from it. Because of the wave nature of sound propagation, phenomena such as refraction, diffraction, diffusion, reflection, reverberation and interference occur. The wide spectrum of sound frequencies that range from infrasounds through acoustic sounds to ultrasounds, also introduces difficulties, as different spectrum components have different penetration properties through the medium. Consequently, SSL is a complex computation problem and development of robust sound localization techniques calls for different approaches, including multisensor schemes, nullsteering beamforming and time-difference arrival techniques. The book offers a rich source of valuable material on advances on SSL techniques and their applications that should appeal to researches representing diverse engineering and scientific disciplines.

\title{
How to reference
}

In order to correctly reference this scholarly work, feel free to copy and paste the following:

J.E. Lugo, R. Doti and J. Faubert (2011). The Impact of Stochastic and Deterministic Sounds on Visual, Tactile and Proprioceptive Modalities, Advances in Sound Localization, Dr. Pawel Strumillo (Ed.), ISBN: 978-953-307224-1, InTech, Available from: http://www.intechopen.com/books/advances-in-sound-localization/the-impactof-stochastic-and-deterministic-sounds-on-visual-tactile-and-proprioceptive-modalities

\section{INTECH}

open science | open minds

\section{InTech Europe}

University Campus STeP Ri

Slavka Krautzeka 83/A

51000 Rijeka, Croatia

Phone: +385 (51) 770447

Fax: +385 (51) 686166

www.intechopen.com

\section{InTech China}

Unit 405, Office Block, Hotel Equatorial Shanghai

No.65, Yan An Road (West), Shanghai, 200040, China

中国上海市延安西路65号上海国际贵都大饭店办公楼405单元

Phone: +86-21-62489820

Fax: $+86-21-62489821$ 
(C) 2011 The Author(s). Licensee IntechOpen. This chapter is distributed under the terms of the Creative Commons Attribution-NonCommercialShareAlike-3.0 License, which permits use, distribution and reproduction for non-commercial purposes, provided the original is properly cited and derivative works building on this content are distributed under the same license. 